\title{
Abstract \\ Assessment of knowledge about hypertension and life style factors that might contribute to hypertension among school teachers in Nuwaragam Palatha-East educational division, Anuradhapura district \\ Wijayathunge LADNL ${ }^{1}$, Hettiaratchi $\mathrm{UPK}^{2 *}$ \\ ${ }^{1}$ Department of Allied Health Sciences, Faculty of Medical Sciences, University of Sri Jayewardenepura \\ ${ }^{2}$ Department of Biochemistry, Faculty of Medical Sciences, University of Sri Jayewardenepura
}

\begin{abstract}
Background

Hypertension is one of the important modifiable risk factors of cardiovascular diseases which have a high mortality in the world. Prevalence of hypertension in Sri Lanka (2014) was 21.2\%. Studies indicate that school teachers are vulnerable to develop hypertension due to work related risk.

\section{Methods}

This was a descriptive cross-sectional study, aimed to assess the knowledge about hypertension and life style factors that might contribute to hypertension among school teachers in Nuwaragam Palatha-East educational division, Anuradhapura. Cluster sampling technique was used to recruit 397 teachers and self administered questionnaire was used.

\section{Results}

Although, most of the subjects (59.9\%) had poor knowledge on hypertension, majority had positive attitudes towards prevention of hypertension. Most of the teachers had following factors and life style behaviors that might contribute to development of hypertension, i.e., $57.8 \%$ have family history of hypertension, $51.6 \%$ were obese, $22.2 \%$ were overweight, $50.9 \%$ never do regular physical activities (exercises for at least 30 minutes/day), $45.7 \%$ male teachers consume alcohol, $19.1 \%$ use non steroidal anti inflammatory drugs, $47.4 \%$ use salt more than the recommended amount/day ( $5 \mathrm{~g} /$ full tea spoon), $27.2 \%$ take $<2$ types of vegetables/day, $84.9 \%$ take $<2$ types of fruits/day and no one takes green leaves every day/week. Most of them have moderate level of satisfaction regarding their life events.

Conclusion

As the level of knowledge on hypertension is poor, there is an urgent need for health education sessions and screening programmes for teachers in Sri Lanka, to prevent hypertension becoming a burden in the country.
\end{abstract}

Key words: Knowledge; Life style factors; Hypertension; School Teachers; Anuradhapura district; Nuwaragam PalathaEast educational division

Copyright: (C) 2015 Wijayathunge LADNL et al. This is an open access article distributed under the Creative Commons Attribution License, which permits unrestricted use, distribution, and reproduction in any medium, provided the original work is properly cited.

\footnotetext{
* Correspondence : kumari31@yahoo.com
}

Cite this abstract as: Wijayathunge LADNL, Hettiaratchi UPK. Assessment of knowledge about hypertension and life style factors that might contribute to hypertension among school teachers in Nuwaragam Palatha-East educational division, Anuradhapura district. Anuradhapura Medical Journal 2015;9 (2Supp):S19.

DOI: http://dx.doi.org/10.4038/amj.v9i2Supp.7568 


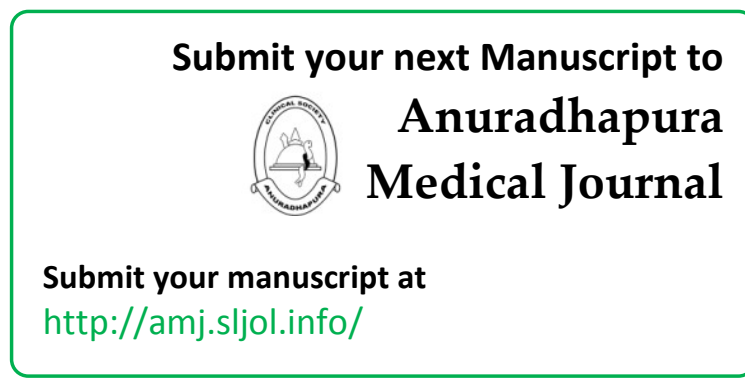

